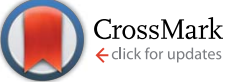

Cite this: RSC Adv., 2016, 6, 55312

\title{
Design of PNIPAAM covalently grafted on halloysite nanotubes as a support for metal-based catalysts $\uparrow$
}

\author{
M. Massaro, ${ }^{a}$ V. Schembri, ${ }^{a}$ V. Campisciano, ${ }^{a}$ G. Cavallaro, ${ }^{b}$ G. Lazzara, ${ }^{\text {tb }}$ S. Milioto, ${ }^{b}$
} R. Noto, ${ }^{a}$ F. Parisi ${ }^{b}$ and S. Riela*a

A thermo-responsive polymer such as poly( $\mathrm{N}$-isopropylacrylamide) (PNIPAAM) was covalently grafted on the external surface of halloysite nanotubes (HNTs) by means of microwave irradiation. This nanomaterial was used as a support and stabilizer for palladium nanoparticles. The obtained HNTPNIPAAM/PdNPS was characterized by means of TGA, SEM, EDS and TEM analyses. The palladium content of the catalyst was estimated to be $0.4 \mathrm{wt} \%$. The stability of the catalytic material at different temperatures (below and above the PNIPAAM lower critical solution temperature) was tested in the Suzuki reaction under microwave irradiation. In addition, TEM analysis after five consecutive runs was performed. The catalyst showed a good catalytic activity toward the Suzuki cross-coupling reaction between phenylboronic acid and several aryl halides in aqueous media under microwave irradiation and low palladium loading $(0.016 \mathrm{~mol} \%, 8 \mu \mathrm{g}$ of $\mathrm{Pd})$. Turnover numbers (TONs) and frequencies (TOFs) up to 6250 and $37500 \mathrm{~h}^{-1}$, respectively, were reached. The catalyst was easily separated from the reaction mixture by centrifugation and reused for five consecutive cycles with a small drop in its catalytic activity.

Received 9th March 2016 Accepted 31st May 2016

DOI: $10.1039 / c 6 r a 06337 c$

www.rsc.org/advances performance have been reported. ${ }^{13-15}$ Gold nanoparticles (NPs) were successfully loaded on halloysite ${ }^{\mathbf{1 6}}$ and the obtained $\mathrm{Au} /$ HNTs hybrid showed high catalytic activity for solvent-free oxidation of benzyl alcohol. ${ }^{17}$

Using clays with tube morphology was beneficial for improving dispersibility and photocatalytic activity of $\mathrm{TiO}_{2}$ NPs. ${ }^{18,19}$ Yang et al. reported palladium NPs deposited on HNTs for hydrogenation of styrene with enhanced catalytic activity. ${ }^{20}$

Recently, we reported the synthesis of novel palladium-based catalytic systems using halloysite nanotubes modified with imidazolium or triazolium moieties as supports for PdNPs and we successfully employed these supported catalysts in the Suzuki reaction under microwave irradiation. ${ }^{21,22}$

Stimuli-responsive polymer materials are playing an increasingly important role in catalysis, since catalytic reactions can be controlled by tuning appropriate stimuli.

Poly( $N$-isopropylacrylamide) (PNIPAAM) hydrogel is one of the most popular thermoresponsive polymers known. It shows a dramatic volume phase transition at the lower critical solution temperature (LCST) around $32{ }^{\circ} \mathrm{C}$, above which it undergoes dehydration and phase separation. The swelling of PNIPAAM hydrogels is mainly due to changes in the hydrogen bonding of the PNIPAAM network with water molecules. At a temperature above LCST, some of the hydrogen bonds are dissociated, and the hydrophobic interactions among the hydrophobic groups in the PNIPAAM network are dominant. ${ }^{23}$ This causes the release of water molecules trapped in the network, causing the hydrogel collapse. PNIPAAM due to its thermo-responsive nature can be loaded or combined with metal particles in order to prepare
${ }^{a}$ Dipartimento STEBICEF, sez. Chimica, Università degli Studi di Palermo, Viale delle Scienze, Ed. 17, 90128, Palermo, Italy.E-mail: serena.riela@unipa.it

${ }^{b}$ Dipartimento di Fisica e Chimica, Università degli Studi di Palermo, Viale delle Scienze, Ed. 17, 90128, Palermo, Italy. E-mail: giuseppe.lazzara@unipa.it

$\dagger$ Electronic supplementary information (ESI) available. See DOI: $10.1039 / \mathrm{c} 6 \mathrm{ra06337c}$ 


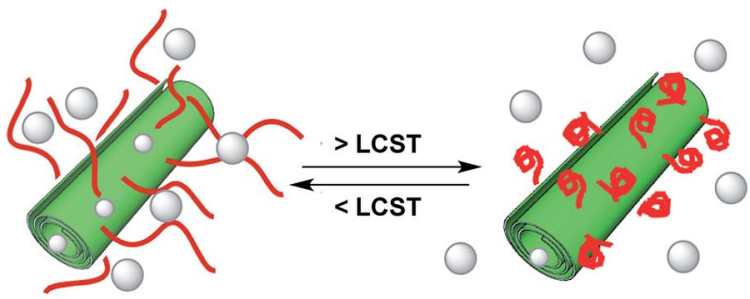

HNT-PNIPAAM/PdNPs

Fig. 1 Cartoon representation of swelling behaviour of HNT-PNIPAAM/PdNPs.

"smart catalysts" that show improved catalytic activities by adjusting the temperature below or above the LCST. In this context several PNIPAAM based catalysts employed for the hydrodechlorination of 4-chlorophenol, the reduction of 4nitrophenol or catalytic wet hydrogen peroxide oxidation of phenol are reported. ${ }^{24}$ Moreover, since PNIPAAM can selectively provide hydrophilic or hydrophobic nano-environments by simply changing the temperature of the reaction medium, it is possible to carry out Suzuki reaction in water under green conditions using PdNPs supported on this polymer. For example, PNIPAM-co-4-vinylpyridine support was used for palladium immobilization and used in Suzuki cross-coupling with excellent results without Pd leaching. ${ }^{25}$

To best of our knowledge, up to date only few examples on halloysite nanotubes dispersed in PNIPAAM aqueous solution are reported. ${ }^{26} \mathrm{HNT} / \mathrm{PNIPAAM}$ hydrogels were used as photocatalysts $^{27}$ as well as catalyst supports for palladium NPs in the Suzuki reaction. ${ }^{28}$

Herein we report an efficient strategy to prepare HNTs-based catalyst through direct chemical grafting with stimuliresponsive polymer (PNIPAAM) coordinating PdNPs (Fig. 1). The HNT-PNIPAAM/PdNPs was tested as catalyst in the Suzuki reaction under microwave irradiation. The use of microwave irradiation provides a rapid, direct and consistent energy for the cross coupling reactions, therefore it had beneficial effects on the efficiency of the Suzuki reactions increasing the reaction rates.

\section{Results and discussion}

The HNT-PNIPAAM material was synthesized by randomly covalent grafting of the carboxylic acid-terminated PNIPAAM to halloysite nanotubes as previous reported by us (Scheme 1). ${ }^{29}$ Furthermore, in order to reduce reaction time, we performed the same reaction under microwave irradiation for $1 \mathrm{~h}$, at temperature of $50{ }^{\circ} \mathrm{C}$, using $\mathrm{CH}_{2} \mathrm{Cl}_{2}$ as solvent. After work up, the obtained nanomaterial was analysed by thermogravimetric analysis (TGA) which confirmed the formation of HNT-PNIPAAM nanomaterial and allowed to calculate the loading percentage of PNIPAAM attached on the HNT surface, which was $13 \mathrm{wt} \%$. It is noteworthy that just $1 \mathrm{~h}$ of $\mathrm{MW}$ irradiation is enough to obtain a loading of polymer on the HNT external

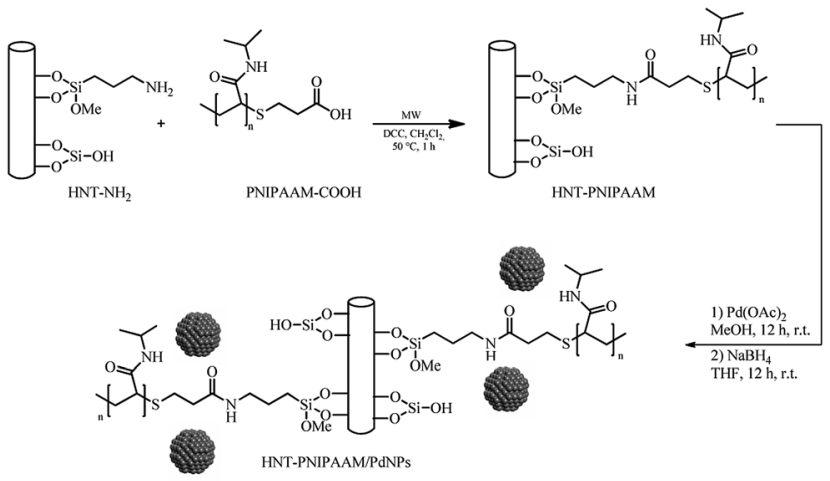

Scheme 1 Schematic representation of the synthesis of HNT-PNIPAAM/PdNPs catalyst.

surface that approaches to the previously reported by $\mathrm{us}^{29}$ in traditional conditions, which take $72 \mathrm{~h}$.

The immobilization of palladium nanoparticles on the support was achieved by solubilizing $\mathrm{Pd}(\mathrm{OAc})_{2}$ in a mixture of HNT-PNIPAAM in methanol. The solid material was recovered by filtration and, finally, the $\mathrm{Pd}(\mathrm{II})$ species were reduced to $\mathrm{Pd}^{0}$ with $\mathrm{NaBH}_{4}$ in tetrahydrofuran. The content of palladium nanoparticles was determined by Inductively Coupled PlasmaOptical Emission Spectrometry (ICP-OES) and was estimated as $1.3 \mathrm{wt} \%$.

The presence of palladium species in the structure of the HNT-PNIPAAM/PdNPs catalyst was confirmed by energydispersive X-ray spectroscopy (EDS) obtained from SEM analysis (Fig. 2). EDS experiment was useful to determine the palladium loading of the catalytic system that was of $1.3 \mathrm{wt} \%$ accordingly to ICP-OES analysis.

EDS measurements further confirmed the successful functionalization highlighting the presence of sulfur in the structure of the nanomaterial deriving from the covalently grafted polymer. SEM micrographs (Fig. 3) showed that the characteristic lengths and the tubular shape of HNTs were preserved in the PNIPAAM-HNT/PdNPs catalyst.

According to the TEM images (Fig. 4), HNT-PNIPAAM/PdNPS catalyst shows the typical rod-shaped structure of HNTs with

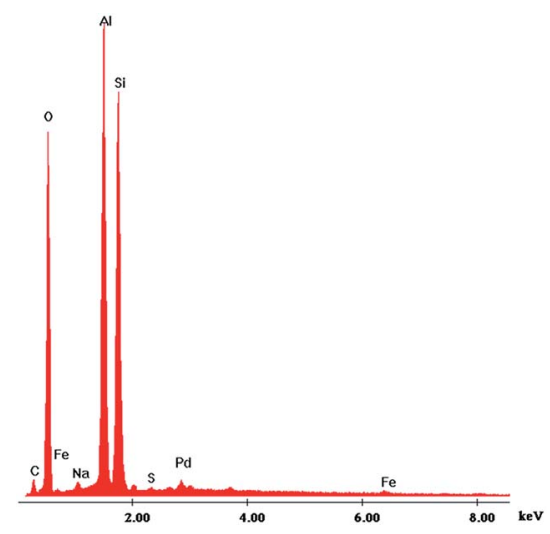

Fig. 2 EDS image of HNT-PNIPAAM/PdNPs catalyst. 


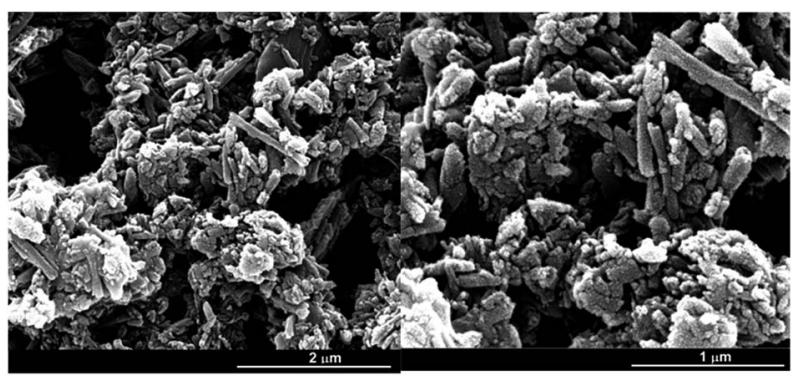

Fig. 3 SEM images of the HNT-PNIPAAM/PdNPs catalyst.
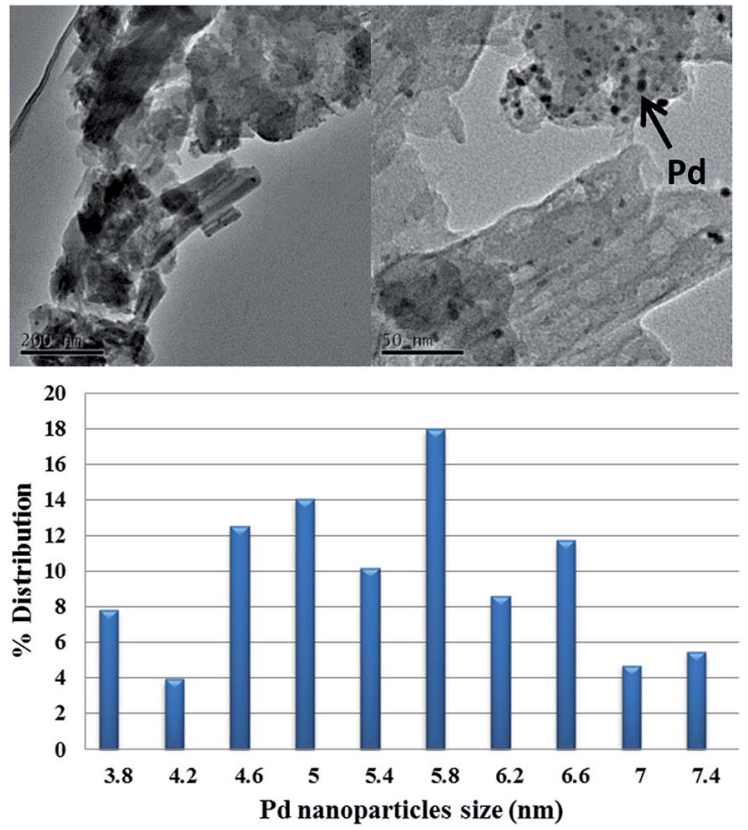

Fig. 4 TEM images of the HNT-PNIPAAM/PdNPs catalyst.

a diameter of about $100 \mathrm{~nm}$ and a length of about $0.7 \mu \mathrm{m}$. The Pd nanoparticles were mainly immobilized on the surface and also anchored to the interlayer of HNTs, having spherical morphologies with diameters of about $5.5 \pm 1.0 \mathrm{~nm}$ (number of counts $(n)=128)$.

The catalytic activity of the HNT-PNIPAAM/PdNPs nanomaterial was evaluated in Suzuki-Miyaura coupling reaction. In particular our studies were focused on determining the influence of temperature and amount of catalyst on the reaction. In order to find the best experimental conditions, the reaction of 4bromoacetophenone with phenylboronic acid was selected as model. Based on our previous studies on Suzuki reaction, with the aim to better solubilize both organic substrate and inorganic base $\left(\mathrm{K}_{2} \mathrm{CO}_{3}\right)$, we carried out the catalytic tests in a water/ ethanol mixture $(50 \% \mathrm{v} / \mathrm{v})$.

As shown in Table 1, excellent results were obtained in all cases, and no byproducts were detected. Thus, the conversions reported correspond to the reaction yields.

To verify the HNT-PNIPAAM/PdNPs stability and its thermoresponsive behaviour, the title Suzuki reaction was carried out
Table 1 Optimization of experimental conditions for Suzuki crosscoupling reaction under microwave irradiation

\begin{tabular}{lc}
\hline \\
Entry \\
1
\end{tabular}

${ }^{a}$ Determined by ${ }^{1} \mathrm{H}$-NMR (Fig. S1-S4). ${ }^{b}$ Reaction conditions: $\mathrm{H}_{2} \mathrm{O} / \mathrm{EtOH}$ (1:1) $1.2 \mathrm{~mL}$, catalyst $0.016 \mathrm{~mol} \%, 2 \mathrm{mg}$.

in a different temperatures ranging between 25 and $120{ }^{\circ} \mathrm{C}$, under microwave irradiation. Catalyst was recovered by centrifugation and was reused in the same reaction for five runs (Fig. 5). When recycling tests were carried out at temperature above the LCST, catalyst afforded the desired biphenyl-4acetophenone product in good yields after four runs ranging from 99 to $77 \%$. However, in the 5 th run we observed a decreased yield (50\%). The amount of Pd nanoparticles released into solution during the reaction was determined by ICP-OES, which was less than $1 \mathrm{wt} \%$. The absence of leaching of palladium has been attributed to a "release and catch" mechanism. ${ }^{30}$ It has been proposed that Suzuki cross-coupling can involve the presence of soluble palladium species (leaching) arising from the surface of the catalyst. However, after completion of the catalytic process, palladium is redeposited onto the support avoiding the leaching in solution.

To confirm the above mentioned mechanism additional experiments were carried out. In three parallel reactions (4bromoanisole and phenylboronic acid) the catalyst was filteredoff after $1 \mathrm{~min}$ of microwave irradiation. In the first one, the reaction was stopped and the crude product was analysed by ${ }^{1} \mathrm{H}-$ NMR. In this case we observed a conversion of $96 \%$ of the

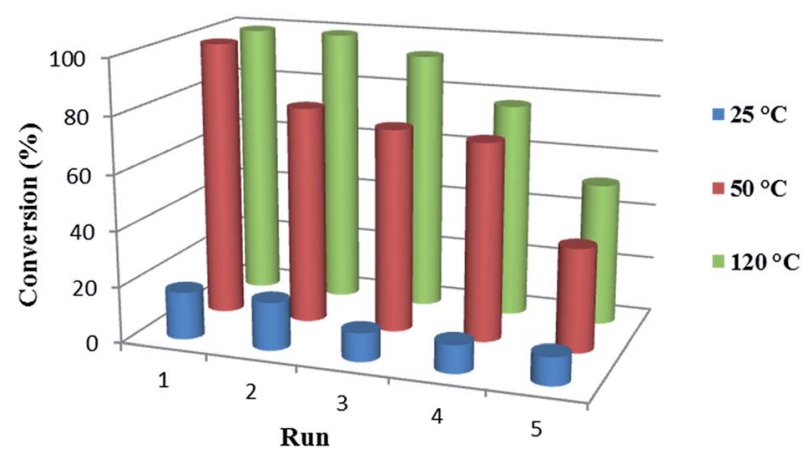

Fig. 5 Effect of temperature on the Suzuki reaction between 4-bromoacetophenone and phenylboronic acid (reaction condition: MW, phenylboronic acid $(0.547 \mathrm{mmol}), 4$-bromoacetophenone $(0.55$ $\mathrm{mmol}), \mathrm{K}_{2} \mathrm{CO}_{3}(0.615 \mathrm{mmol}), \mathrm{EtOH} / \mathrm{H}_{2} \mathrm{O} 1: 1(1.2 \mathrm{~mL})$, catalyst $(0.16$ $\mathrm{mol} \%, 7 \mathrm{mg})$ ). 
correspondent biaryl derivative. The second reaction was quickly filtered still hot (hot filtration) and left to stir (without any catalyst) for further 9 min leading to a total conversion (>99\%). Conversely, the last reaction, which was cooled down to rt prior filtration and reacted for 9 min (cold filtration), showed no extra conversion, actually we observed a decrease (70\%), probably due to the degradation of reactants and products caused by the prolonged microwave irradiation. To further confirm the release and catch mechanism an additional reaction was carried out as follow. The catalyst was treated at $120{ }^{\circ} \mathrm{C}$ for $3 \mathrm{~min}$ under microwave irradiation in a mixture $\mathrm{H}_{2} \mathrm{O} / \mathrm{EtOH} 1: 1$. It was filtered still hot and to the solution obtained were added the reactants (4-bromoanisole and phenylboronic acid) and the Suzuki reaction was performed for $10 \mathrm{~min}$ in the experimental conditions described above. In this way we obtained a $12 \%$ of conversion, confirming the presence of soluble Pd species in solution released from the catalyst at high temperature.

On the contrary, as expected for the thermo-responsive behaviour (LCST $<32{ }^{\circ} \mathrm{C}$ ), at $25{ }^{\circ} \mathrm{C}$ very low conversions (about $10 \%$ ) were observed and, also in this case ICP-OES analysis showed no palladium leaching during the course of Suzuki reaction. Interestingly, when the catalyst was reused, after recovering, in the same reaction at $50{ }^{\circ} \mathrm{C}$, an increase in the conversion $(34 \%)$ was observed.

The difference of catalytic activity of HNT-PNIPAAM/PdNPs at different temperatures can be attributed to the thermoresponsive behaviour of PNIPAAM grafted onto halloysite surface. At room temperature, the PNIPAAM chains should adopt an extended conformation forming a hydrophilic nanoenvironment. Therefore, the Suzuki reaction, with hydrophobic substrates, such as 4-bromoacetophenone, is unsatisfactory and the yields are low (Fig. 5). Above the LCST the PNIPAAM chains collapse to form a dense layer covering HNT surface; the grafted PNIPAAM brushes become hydrophobic, facilitating the mass-transfer of hydrophobic substrates and promoting the cross coupling process. ${ }^{31}$ As a result, we observed higher conversions at temperature above the LCST.

Such temperature-responsive behaviour was reversible, as the recycled catalyst showed the same activity and thermoresponsive behaviour. The influence of temperature in catalytic process was already reported in literature for PNIPAAM@SBA systems. ${ }^{32}$

To explain the drop of catalytic activity after five runs carried out above the LCST, we performed TEM analysis keeping in mind that $\mathrm{Pd}^{0}$ nanoparticles have the tendency to agglomerate during the catalytic cycles. This experiment (Fig. 6) revealed agglomeration of $\mathrm{Pd}$ nanoparticles (mean diameter $6.6 \pm 1.2$ $\mathrm{nm}, n=128$ ) on the surface of HNTs, furnishing a possible explanation for the drop of the catalytic activity due to the reduction in the surface area and saturation of the coordination sites. ${ }^{33}$ This could be explained by the Ostwald ripening favoured under the harsh catalytic reaction conditions used. After catalytic process, the small soluble Pd species leached by the catalyst surface, as above mentioned, are redeposited on the support forming larger and less reactive Pd clusters. ${ }^{34}$

In the last years the use of water as reaction medium for coupling reaction catalyzed by heterogeneous palladium
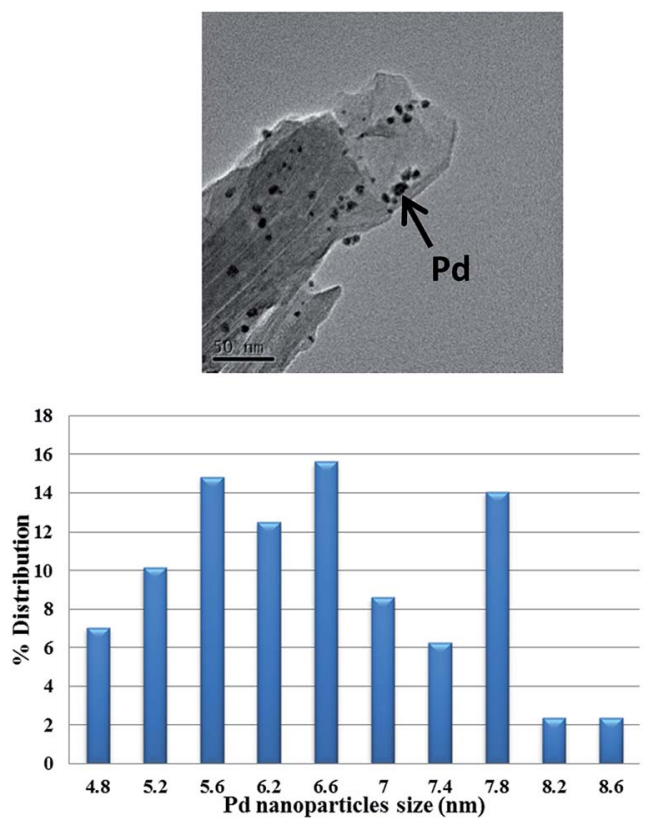

Fig. 6 TEM image of HNT-PNIPAAM/PdNPs material after five catalytic runs.

catalyst has received considerable attention due to the rising concerns for the environment being that water is cheap, environmental friendly, and allows simple separation and catalyst recycling.

With this idea in mind, we decided to perform the Suzuki coupling reaction in water, using $\mathrm{K}_{2} \mathrm{CO}_{3}$ as base and under microwave irradiation. An accurate screening of the reaction condition was carried out choosing the coupling between 4bromoacetophenone and phenylboronic acid as reaction model.

In order to obtain the best combination the influence of different parameters, namely reaction time, temperature, catalyst concentration as well as the addition of a transfer phase additive (Table 2), were examined. In the most of the cases the conversions reported in Table 2 correspond to yields. The better results were obtained with an irradiation time of $10 \mathrm{~min}$ and at $120{ }^{\circ} \mathrm{C}$ (entry 4). It is worth to note that even though $15 \mathrm{~min}$ of irradiation lead to higher conversions, however we observed a decrease in the yield (entry 5). Prolonged irradiation, indeed, causes decreased in conversions (entries 3-8) and yields (entry 5) probably due to the formation of by-products such as the homocoupling and the dehalogenation products or degradation of both reactants and products. ${ }^{35}$

The use of a transfer phase additive, such as tetrabutylammonium bromide (TBAB), even in low amount, accelerated the reaction (entry 6) since it facilitated the dissolution of the organic reactants in the water phase. Then we decided to further reduce the amount of catalyst down to $0.016 \mathrm{~mol} \%$, obtaining quantitative conversion (entry 9).

The scope of Suzuki cross coupling reactions was investigated using phenylboronic acid and a broad range of aryl halides under the optimized conditions. The reactions of differently substituted aryl halides and phenylboronic acid were 
Table 2 Optimization of experimental conditions for Suzuki crosscoupling reaction under microwave irradiation

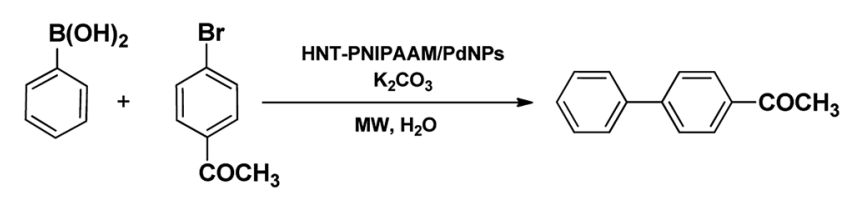

\begin{tabular}{llllll} 
Entry & Pd (mol\%) & Additive & $\begin{array}{l}t \\
(\mathrm{~min})\end{array}$ & $T\left({ }^{\circ} \mathrm{C}\right)$ & Conv. $^{a}(\%)$ \\
\hline 1 & 0.16 & - & 10 & 70 & 20 \\
2 & 0.16 & - & 15 & 70 & 36 \\
3 & 0.16 & - & 30 & 70 & 20 \\
4 & 0.16 & - & 10 & 120 & 67 \\
5 & 0.16 & - & 15 & 120 & $85(55)^{c}$ \\
6 & 0.16 & $\mathrm{TBAB}^{b}$ & 2 & 120 & 90 \\
7 & 0.16 & $\mathrm{TBAB}^{b}$ & 10 & 120 & $>99$ \\
8 & 0.16 & $\mathrm{TBAB}^{b}$ & 15 & 120 & 93 \\
9 & 0.016 & $\mathrm{TBAB}^{b}$ & 10 & 120 & $>99$
\end{tabular}

${ }^{a}$ Determined by ${ }^{1} \mathrm{H}-\mathrm{NMR}$ (Fig. S5-S13). ${ }^{b}$ Reaction conditions: $\mathrm{H}_{2} \mathrm{O}(1.2$ $\mathrm{mL}$ ), tetrabutylammonium bromide $0.05 \mathrm{mmol} .{ }^{c}$ Yield (\%).

carried out in presence of $\mathrm{K}_{2} \mathrm{CO}_{3}$ (1.12 eq.), TBAB as additive (0.05 mmol) and $0.016 \mathrm{~mol} \%(0.7 \mathrm{mg})$ of HNT-PNIPAAM/PdNPs catalyst using water as solvent. The reactions were heated at $120{ }^{\circ} \mathrm{C}$ for $10 \mathrm{~min}$ under microwave irradiation.

Conversions were high, ranging from $73 \%$ to $99 \%$; in all case no byproducts were detected and the conversions reported in Table 3 correspond to yields.

The less reactive aryl chlorides gave no or very low conversions (entries 9 and 10) under the same reaction conditions.

The obtained good results could be due, once again, to the thermo-responsive behavior of PNIPAAM.

Table 3 Suzuki cross-coupling reaction of phenylboronic acid with various aryl halides under optimized reaction conditions under microwave irradiation

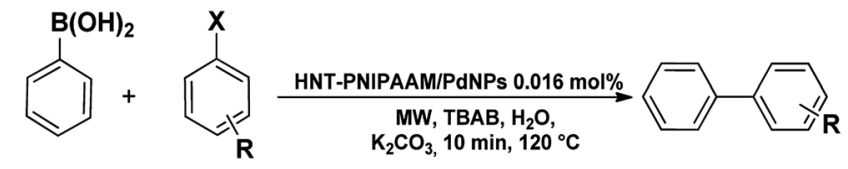

\begin{tabular}{lllll}
\hline Entry & Ar-X & Conv. $^{a}(\%)$ & TON $^{b}$ & TOF $^{c}\left(\mathrm{~h}^{-1}\right)$ \\
\hline 1 & 4-Bromoacetophenone & $>95$ & 6250 & 37500 \\
2 & 4-Iodoanisole & 85 & 5310 & 31870 \\
3 & 4-Bromobenzaldheyde & 94 & 5880 & 35250 \\
4 & 4-Bromoanisole & 85 & 5310 & 31880 \\
5 & 3-Bromobenzaldheyde & 82 & 5130 & 30750 \\
6 & 3-Bromoanisole & 64 & 4000 & 24000 \\
7 & 4-Iodoacetophenone & 94 & 5880 & 35250 \\
8 & 2-Iodotoluene & 73 & 4560 & 27380 \\
9 & 4-Chlorobenzaldheyde & - & - & - \\
10 & 1-Chloro-4-nitrobenzene & 4 & 250 & 1500
\end{tabular}

${ }^{a}$ Determined by ${ }^{1} \mathrm{H}-\mathrm{NMR}$ (Fig. S13-S21). ${ }^{b}$ Turnover number (TON) calculated as moles of substrate converted/moles of palladium. ${ }^{c}$ Turnover frequency (TOF) calculated as TON/hours.
The grafted PNIPAAM brushes, indeed, become hydrophobic and collapse on the HNT surface at temperature above the LCST, and therefore could provide a nano-environment for hydrophobic guest molecules in water.

These results demonstrate the good catalytic activity of HNTPNIPAAM/PdNPs in microwave promoted Suzuki cross-coupling reaction at $120^{\circ} \mathrm{C}$ with turnover numbers (TONs) up to 6250 and turnover frequencies (TOFs) up to $37500 \mathrm{~h}^{-1}$.

The recycling ability of this novel supported palladium catalyst was also investigated. For this purpose the microwave assisted Suzuki cross coupling reaction of 4-bromoacetophenone with phenylboronic acid under the optimized reaction conditions was studied. Because of the very small amount of catalyst used $(0.7 \mathrm{mg})$, we carried out recycling investigations using catalyst at $0.07 \mathrm{~mol} \%$ (3 mg).

After each cycle, the catalyst was recovered by centrifugation, washed with diethyl ether and water and directly used for the next cycle. The catalyst showed to be recyclable for five consecutive times. The activity slightly dropped in the run 3 yielding $85 \%$ conversion and further dropped in run 5 to $70 \%$ (Fig. 7). In order to determine the degree of leaching of the metal from the heterogeneous catalyst, the palladium content of the supernatant, after each cycle of washing, was determined by ICP-OES and no leaching was detected. Thus the catalytic activity dropped to some extent after the 5th cycle and it could be ascribed to agglomeration of Pd nanoparticles on the surface of HNT-PNIPAAM, as above mentioned.

It could be noteworthy to compare the performance of the HNT-PNIPAAM/Pd catalyst with other supported Pd nanoparticle catalysts developed for Suzuki cross coupling reactions. Several studies reported Pd nanoparticles stabilized by hybrid polymeric materials used as catalysts in Suzuki reactions. ${ }^{36-38}$ Among them Pd nanoparticles were stabilized by polyvinyl pyrrolidone (PVP), polystyrene-polyethylene oxide and poly(amido-amine) dendrimers as well as other polymers. ${ }^{38,39}$

However some nanocatalysts have shown limited catalytic activity such as lower product yields using low catalyst loadings, no recyclability and low TON and TOF values. ${ }^{38}$ For example, catalysts based on Pd supported on PVP afforded the Suzuki products, under microwave irradiation, in $75-79 \%$ and $43-87 \%$ yields for aryl iodides and bromides, respectively. ${ }^{40}$

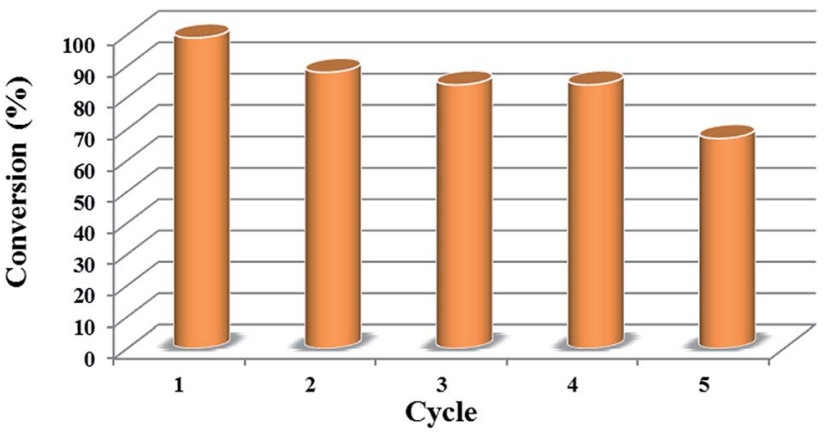

Fig. 7 Recycling investigations (reaction condition: MW, $10 \mathrm{~min}, 120$ ${ }^{\circ} \mathrm{C}$, phenylboronic acid $(0.547 \mathrm{mmol}), 4$-bromoacetophenone $(0.550$ $\mathrm{mmol}), \mathrm{K}_{2} \mathrm{CO}_{3}(0.615 \mathrm{mmol}), \mathrm{H}_{2} \mathrm{O}(1.2 \mathrm{~mL}), \mathrm{TBAB}(0.05 \mathrm{mmol})$, catalyst (0.07 mol\%, $3.0 \mathrm{mg})$ ). 
Furthermore the Suzuki reactions of aryl iodides and bromides were carried out by microwave irradiation using higher $\mathrm{Pd} /$ poly $(N, N$-dihexylcarbodiimide) catalyst loadings (0.5 mol\%) that could be recycled up to five times with slightly decreased activity. ${ }^{41}$ Recently, thermo-responsive polymers have been used as both stabilizer and support for the Pd nanoparticles and resulted in highly recyclable catalysts for the Suzuki coupling of several aryl halide and phenylboronic acid. ${ }^{28}$ On the basis of these examples, it can be concluded that the current HNTPNIPAAM/Pd material provides highly stable and efficient catalysts for cross coupling reactions as compared to other Pd nanoparticles stabilized by polymeric molecules.

\section{Experimental section}

All reagents needed were used (Aldrich) without further purification.

Halloysite (from Sigma-Aldrich) has an average tube diameter of $50 \mathrm{~nm}$ and inner lumen diameter of $15 \mathrm{~nm}$. Typical specific surface area of this halloysite is $65 \mathrm{~m}^{2} \mathrm{~g}^{-1}$; pore volume of $1.25 \mathrm{~mL} \mathrm{~g}^{-1}$; refractive index 1.54; and specific gravity $2.53 \mathrm{~g}$ $\mathrm{cm}^{-3}$. HNT-NH $\mathrm{H}_{2}$ was synthesized as previously reported. ${ }^{29}$

Thermogravimetric analyses were performed by a Q5000 IR apparatus (TA Instruments) under a nitrogen flow of $25 \mathrm{~cm}^{3}$ $\mathrm{min}^{-1}$ for the sample and $10 \mathrm{~cm}^{3} \mathrm{~min}^{-1}$ for the balance. The weight of each sample was $c a .10 \mathrm{mg}$. The measurements were carried out by heating the sample from room temperature to $900{ }^{\circ} \mathrm{C}$ at a rate of $10^{\circ} \mathrm{C} \min ^{-1}$.

The microscope ESEM FEI QUANTA 200F with EDX probe was used to study the morphology and elemental analysis of the HNT-PNIPAAM. Before each experiment, the sample was coated with gold in argon by means of an Edwards Sputter Coater S150A to avoid charging under electron beam.

MW-assisted syntheses were carried out with a CEM DISCOVER monomode system in closed vessel.

${ }^{1} \mathrm{H}$ NMR spectra were recorded at $300 \mathrm{MHz}$ in $\mathrm{CDCl}_{3}$ solutions at room temperature on a Bruker $300 \mathrm{MHz}$ spectrometer.

TEM micrographs were acquired with a Jeol JEM 2100 microscope operating at $200 \mathrm{kV}$. A drop of each dispersion has been deposited in a $3 \mathrm{~mm}$ nickel grid holey carbon coated (Taab). The grid was dried overnight before observation. No coating was applied for TEM observation.

Inductively Coupled Plasma-Optical Emission Spectrometry (ICP-OES) analyses were carried out with an Optima 2100, Perkin Elmer, equipped with an auto sampler model AS-90. Analyses were conducted using a calibration curve, obtained by dilution (range: $0-10 \mathrm{ppm}$ ) of palladium standard solution for ICP-OES analyses. The operative wavelengths were 340.458 and $363.470 \mathrm{~nm}$.

\section{Synthesis of HNT-PNIPAAM via MW}

PNIPAAM (94 mg, 10 equiv.), N,N-dicyclohexylcarbodiimide (DCC) (88 mg, 10 equiv.) and $\mathrm{CH}_{2} \mathrm{Cl}_{2}(1 \mathrm{~mL})$ were placed in a microwave test tube provided with a cap. The mixture was stirred under argon atmosphere, at room temperature for 10 min. Afterward HNT- $\mathrm{NH}_{2}(500 \mathrm{mg})$ was quickly added. Then, the mixture was inserted into microwave apparatus setting a temperature of $50{ }^{\circ} \mathrm{C}$ and under constant stirring for $60 \mathrm{~min}$, then filtered under reduced pressure, washed whit $\mathrm{H}_{2} \mathrm{O}$ and dried overnight under reduced pressure at room temperature.

\section{Typical procedure for the synthesis of the palladium catalysts}

In a $50 \mathrm{~mL}$ round bottom flask were placed HNT-PNIPAAM (100 $\mathrm{mg}), \mathrm{Pd}(\mathrm{OAc})_{2}(10 \mathrm{mg}, 0.0445 \mathrm{mmol})$ and $\mathrm{MeOH}(20 \mathrm{~mL})$. The obtained dispersion was sonicated for $1 \mathrm{~min}$ and was stirred for $18 \mathrm{~h}$ at room temperature. The mixture was filtered under reduced pressure, washed three times with $20 \mathrm{~mL} \mathrm{MeOH}$ and dried under reduced pressure at room temperature. The material was re-suspended in THF $(20 \mathrm{~mL})$ and to this suspension $\mathrm{NaBH}_{4}$ (10 mg, $0.264 \mathrm{mmol}$ ) as a reducing reagent was added. The suspension was stirred at room temperature for $18 \mathrm{~h}$. $\mathrm{MeOH}(10 \mathrm{~mL})$ was added to destroy the $\mathrm{NaBH}_{4}$ residual, then filtered under reduced pressure, washed whit $20 \mathrm{~mL}$ of $\mathrm{MeOH}$ and $20 \mathrm{~mL}$ of THF and then dried overnight under reduced pressure at room temperature.

\section{Typical procedure for the Suzuki reaction via microwave irradiation}

HNT-PNIPAAM/PdNPs catalyst (0.16 $\mathrm{mol} \%$ or $0.016 \mathrm{~mol} \%)$, phenylboronic acid (65 mg, $0.547 \mathrm{mmol}$ ), $\mathrm{K}_{2} \mathrm{CO}_{3}$ (84 mg, 0.61 $\mathrm{mmol})$, aryl bromide $(0.550 \mathrm{mmol}), \mathrm{H}_{2} \mathrm{O} /$ EtOH $(50 \% \mathrm{v} / \mathrm{v})(1.2$ $\mathrm{mL}$ ) or water $(1.2 \mathrm{~mL})$ were placed in a microwave pressurized test tube provided with a sealed cap. The mixture was inserted into microwave apparatus setting a temperature of $120{ }^{\circ} \mathrm{C}(14$ $\mathrm{W})$, under constant stirring for $10 \mathrm{~min}$; the solvent was then removed under reduced pressure and the residue was checked by ${ }^{1} \mathrm{H}$ NMR to calculate conversion.

\section{Recyclability of the catalyst}

HNT-PNIPAAM/PdNPs catalyst (3 mg, $0.07 \mathrm{~mol} \%$ ), phenylboronic acid ( $65 \mathrm{mg}, 0.547 \mathrm{mmol}$ ), $\mathrm{K}_{2} \mathrm{CO}_{3}$ (84 mg, $0.615 \mathrm{mmol}$ ), 4-bromoacetophenone $(0.55 \mathrm{mmol})$, a mixture of $\mathrm{H}_{2} \mathrm{O} / \mathrm{EtOH}$ $(1: 1)(1.2 \mathrm{~mL})$ or water $(1.2 \mathrm{~mL})$ were placed in a microwave pressurized test tube provided with a sealed cap. The mixture was inserted into microwave apparatus setting a temperature of $120{ }^{\circ} \mathrm{C}(14 \mathrm{~W})$, under constant stirring for $10 \mathrm{~min}$. The reaction mixture was then centrifuged and the supernatant was decanted; the residual solid was washed with $\mathrm{CH}_{2} \mathrm{Cl}_{2}, \mathrm{Et}_{2} \mathrm{O}$ and water. HNT-PNIPAAM/PdNPs was dried and reused.

\section{Conclusions}

In conclusion, we have covalently linked PNIPAAM thermoresponsive polymer on halloysite external surface by a simple synthetic route. The HNT-PNIPAAM nanomaterial was used as support and stabilizer of palladium nanoparticles. EDS analysis and TEM images confirmed the presence of Pd on the support, whereas SEM images showed that upon palladium immobilization the tubular shape of halloysite was preserved. The catalyst stability at varying temperatures in the Suzuki reaction was tested. TEM analysis after five consecutive runs showed Pd agglomeration. 
The catalytic performance of this hybrid material was tested in Suzuki cross-coupling reaction of aryl halides with phenylboronic acid in aqueous media under microwave irradiation. The good catalytic activity was also demonstrated by the low amount of catalyst employed (just $0.016 \mathrm{~mol} \%, 2.34 \mathrm{mg}$ ) and high TON and TOF values. Furthermore, the catalyst showed good recyclability for five cycles with negligible Pd leaching.

\section{Acknowledgements}

The work was financially supported by the University of Palermo, FIRB 2012 (prot. RBFR12ETL5), PON-TECLA (PON03PE_00214_1). TEM images were provided by Centro Grandi Apparecchiature-UniNetLab - Università di Palermo funded by P.O.R. Sicilia 2000-2006, Misura 3.15 Quota Regionale. The authors would thanks Dr A. Pettignano (University of Palermo) for ICP-OES analyses.

\section{Notes and references}

1 M. Liu, Z. Jia, D. Jia and C. Zhou, Prog. Polym. Sci., 2014, 39, 1498-1525.

2 M. Massaro, S. Riela, G. Cavallaro, M. Gruttadauria, S. Milioto, R. Noto and G. Lazzara, J. Organomet. Chem., 2014, 749, 410-415.

3 P. Pasbakhsh, G. J. Churchman and J. L. Keeling, Appl. Clay Sci., 2013, 74, 47-57.

4 F. Arcudi, G. Cavallaro, G. Lazzara, M. Massaro, S. Milioto, R. Noto and S. Riela, J. Phys. Chem. C, 2014, 118, 1509515101.

5 V. Vergaro, E. Abdullayev, Y. M. Lvov, A. Zeitoun, R. Cingolani, R. Rinaldi and S. Leporatti, Biomacromolecules, 2010, 11, 820826.

6 M. Kryuchkova, A. Danilushkina, Y. Lvov and R. Fakhrullin, Environ. Sci.: Nano, 2016, 3, 442-452.

7 M. Massaro, C. G. Colletti, R. Noto, S. Riela, P. Poma, S. Guernelli, F. Parisi, S. Milioto and G. Lazzara, Int. J. Pharm., 2015, 478, 476-485.

8 S. Riela, M. Massaro, C. G. Colletti, A. Bommarito, C. Giordano, S. Milioto, R. Noto, P. Poma and G. Lazzara, Int. J. Pharm., 2014, 475, 613-623.

9 Y. Lvov, A. Aerov and R. Fakhrullin, Adv. Colloid Interface Sci., 2014, 207, 189-198.

10 M. R. Dzamukova, E. A. Naumenko, Y. M. Lvov and R. F. Fakhrullin, Sci. Rep., 2015, 5, 10560.

11 S. A. Konnova, I. R. Sharipova, T. A. Demina, Y. N. Osin, D. R. Yarullina, O. N. Ilinskaya, Y. M. Lvov and R. F. Fakhrullin, Chem. Commun., 2013, 49, 4208-4210.

12 M. Massaro, S. Riela, P. Lo Meo, R. Noto, G. Cavallaro, S. Milioto and G. Lazzara, J. Mater. Chem. B, 2014, 2, 77327738.

13 G. S. Machado, O. J. D. Lima, K. J. Ciuffi, F. Wypych and S. Nakagaki, Catal. Sci. Technol., 2013, 3, 1094-1101.

14 A. M. Carrillo and J. G. Carriazo, Appl. Catal., B, 2015, 164, 443-452.

15 C. Li, X. Li, X. Duan, G. Li and J. Wang, J. Colloid Interface Sci., 2014, 436, 70-76.
16 M. Zieba, J. L. Hueso, M. Arruebo, G. Martinez and J. Santamaria, New J. Chem., 2014, 38, 2037-2042.

17 X. Fu, Z. Ding, X. Zhang, W. Weng, Y. Xu, J. Liao and Z. Xie, Nanoscale Res. Lett., 2014, 9, 282.

18 D. Papoulis, S. Komarneni, D. Panagiotaras, E. Stathatos, D. Toli, K. C. Christoforidis, M. Fernández-García, H. Li, S. Yin, T. Sato and H. Katsuki, Appl. Catal., B, 2013, 132133, 416-422.

19 C. Li, J. Wang, S. Feng, Z. Yang and S. Ding, J. Mater. Chem. A, 2013, 1, 8045-8054.

20 Y. Zhang, X. He, J. Ouyang and H. Yang, Sci. Rep., 2013, 3, 2948.

21 M. Massaro, S. Riela, G. Lazzara, M. Gruttadauria, S. Milioto and R. Noto, Appl. Organomet. Chem., 2014, 28, 234-238.

22 M. Massaro, S. Riela, G. Cavallaro, C. G. Colletti, S. Milioto, R. Noto, F. Parisi and G. Lazzara, J. Mol. Catal. A: Chem., 2015, 408, 12-19.

23 M. Shibayama, Y. Suetoh and S. Nomura, Macromolecules, 1996, 29, 6966-6968.

24 Q. Wang, J. Wang, D. Wang, M. Turhong and M. Zhang, Chem. Eng. J., 2015, 280, 158-164.

25 M. C. Hong, M. C. Choi, Y. W. Chang, Y. Lee, J. Kim and H. Rhee, Adv. Synth. Catal., 2012, 354, 1257-1263.

26 G. Cavallaro, G. Lazzara, S. Milioto and F. Parisi, J. Colloid Interface Sci., 2016, 461, 346-351.

27 W. Xing, L. Ni, X. Liu, Y. Luo, Z. Lu, Y. Yan and P. Huo, RSC Adv., 2013, 3, 26334-26342.

28 M. C. Hong, H. Ahn, M. C. Choi, Y. Lee, J. Kim and H. Rhee, Appl. Organomet. Chem., 2014, 28, 156-161.

29 G. Cavallaro, G. Lazzara, M. Massaro, S. Milioto, R. Noto, F. Parisi and S. Riela, J. Phys. Chem. C, 2015, 119, 8944-8951.

30 M. Gruttadauria, F. Giacalone and R. Noto, Green Chem., 2013, 15, 2608-2618.

31 G. Wei, W. Zhang, F. Wen, Y. Wang and M. Zhang, J. Phys. Chem. C, 2008, 112, 10827-10832.

$32 \mathrm{X} . \mathrm{Wu}, \mathrm{X}$. Chen, H. Guan, X. Wang and L. Chen, Catal. Commun., 2014, 51, 29-32.

33 A. R. Siamaki, Y. Lin, K. Woodberry, J. W. Connell and B. F. Gupton, J. Mater. Chem. A, 2013, 1, 12909-12918.

34 J. Durand, E. Teuma and M. Gómez, Eur. J. Inorg. Chem., 2008, 2008, 3577-3586.

35 T. N. Danks and G. Wagner, in Microwave Assisted Organic Synthesis, Blackwell Publishing Ltd, 2009, pp. 75-101, DOI: 10.1002/9781444305548.ch4.

36 C. J. Welch, J. Albaneze-Walker, W. R. Leonard, M. Biba, J. DaSilva, D. Henderson, B. Laing, D. J. Mathre, S. Spencer, X. Bu and T. Wang, Org. Process Res. Dev., 2005, 9, 198-205.

37 P. J. Ellis, I. J. S. Fairlamb, S. F. J. Hackett, K. Wilson and A. F. Lee, Angew. Chem., Int. Ed., 2010, 49, 1820-1824.

38 A. Balanta, C. Godard and C. Claver, Chem. Soc. Rev., 2011, 40, 4973-4985.

39 F. Giacalone, V. Campisciano, C. Calabrese, V. La Parola, Z. Syrgiannis, M. Prato and M. Gruttadauria, ACS Nano, 2016, 10, 4627-4636.

40 D. de Luna Martins, H. M. Alvarez and L. C. S. Aguiar, Tetrahedron Lett., 2010, 51, 6814-6817.

41 Y. Liu, C. Khemtong and J. Hu, Chem. Commun., 2004, 398399. 